\title{
Quo Vadis Hybrid Intelligent Systems: New Trends and Approaches
}

\author{
Ajith Abracham \\ Norwegian University of Science and Technology, \\ Trondheim, Norway \\ ajith.abraham@q2s.ntnu.no
}

\begin{abstract}
We are blessed with the sophisticated technological artifacts and the real world problems are getting more complicated. The emerging need for advanced Hybrid Intelligent Systems (HIS) is currently motivating important research and development work. The integration of different learning/adaptation and optimization techniques, to overcome individual limitations and achieve synergetic effects through hybridization of several intelligent algorithms, has in recent years contributed to a large number of new intelligent systems architectures. This talk presents a quick review of some of the generic hybrid architectures, which have evolved in the HIS community. We further attempt to discuss the importance of these architectures with an emphasis on the best practices for selection and combination of intelligent methods. Several application examples will be presented to demonstrate how such systems could be used for solving real world problems.
\end{abstract}

